\title{
Pansharpening of Multispectral Satellite Images via Lattice Structures
}

\author{
N.H. Kaplan \\ Erzurum Technical University Yakutiye/Erzurum
}

\author{
I. Erer \\ Istanbul Technical University Ayazaga/Istanbul
}

\begin{abstract}
Since satellite images with high spatial and spectral quality are highly desired for remote sensing applications, various algorithms have been developed for the fusion of multispectral and panchromatic images. Wavelet transform based mergers have found enormous interest in the fusion community. This paper introduces undecimated filterbanks with lattice structure and applies them to the pansharpening problem. Multispectral and panchromatic images are decomposed using the developed lattice analysis structure into subbands which are combined by using a predefined fusion rule. The fused image is obtained by the inverse lattice filtering of the fused subbands. Fusion results and quality metrics show that the proposed method can be a good alternative to the other well-known pansharpening methods.
\end{abstract}

\section{General Terms}

Image processing, remote sensing

\section{Keywords}

Image fusion, pansharpening, multispectral images, multiresolution analysis, lattice filters.

\section{INTRODUCTION}

Many remote sensing satellite systems provide both MS (multispectral) images and PAN (panchromatic) images. It is well known that MS images suffer from low spatial resolution, while high spatial resolution PAN images have low spectral quality. However, a high spatial and spectral quality satellite image is desired for remote sensing applications. Pansharpening is a pixel level fusion technique which may be used to address this problem: it combines MS and PAN images to produce an image which is as close as possible to that would be produced by observing the same ground area by a multispectral sensor with the same resolution as the panchromatic sensor [1,2]. The fused image has higher spatial resolution, while it preserves the spectral characteristics of the MS image.

Various techniques for the fusion MS and PAN images have been developed [2-10]. The well-known methods are Brovey transform [2], component substitution based fusion methods such as IHS (Intensity-Hue-Saturation) [4-6] and PCA (Principal Component Analysis) [7]. The Brovey sharpened images show high contrast [2]. Carper et al. used the IHS transformation method to perform pansharpening [4] of SPOT MS image. Chavez et al. used the principal component analysis (PCA) based method for merging LANDSAT MS image and SPOT PAN image [7]. The standard fusion algorithms described above have a wide area of use, because of their relatively simple and time efficient fusion schemes. They are often successful to increase the spatial resolution; however, they tend to distort the spectral information present in the original multispectral image.

An alternative to these methods, are the multiresolution analysis based ones among of which wavelet transform based ones give the best results $[9,10]$. In wavelet transform based substitutive techniques, MS and PAN images are decomposed into subbands through wavelet transform, and the detail information extracted from the detail subbands of the PAN image is injected to the wavelet coefficients of the fused image using a predefined fusion rule, then the inverse wavelet transform is applied to obtain the fused image. However, during the detail injection process, spatial distortions, such as ringing effect or aliasing problems can be seen in the fused image. Such artifacts may be amplified by misregistration between MS and PAN data, especially if the wavelet transform is not shift-invariant as in the case of Mallat's discrete wavelet transform (DWT) algorithm. A number of different wavelet based sharpening techniques which use shift-invariant methods are available, like as the stationary wavelet transform (SWT) [9].

In this paper, a new image fusion algorithm based on the subband decomposition of the source images using undecimated 1-D lattice filter structures is proposed. Lattice filters have applications in a wide area, because of their modularity, low sensitivity and simple test for stability [12, 13]. Lattice structure based two channel quadrature mirror filter (QMF), has both good stopband attenuation and perfect reconstruction properties. It is possible to increase the order of lattice structure based QMF filterbank by adding more lattice layers. The analysis part of the filterbank is achieved by a lattice filter, whereas, the synthesis part is achieved by the inverse filter [12]. The lattice structure defined in [12] does not have shift-invariance property. Therefore, in this paper we propose undecimated lattice decomposition (ULD) and reconstruction (ULR) structures. Then, by the help of ULD, each band of the MS image and PAN image is decomposed into sub images. The sub images are combined using a predefined rule and ULR is used to obtain the fused image.

The rest of the paper is organized as follows. Section 2 describes a background information on 1-D PR Lattice structure. In section 3, proposed undecimated subband decomposition and reconstruction are described and the fusion process is described. The experimental results are given in section 4 and general conclusions are drawn in section 4 .

\section{BACKGROUND ON 1-D LATTICE STRUCTURE}

1-D Filterbank with lattice structure given in [12] consists of two parts: analysis and synthesis sections. In the analysis section the input signal is splatted into low and high pass components and in the synthesis section it is reconstructed from its components [12].

QMFB with lattice structure involves a cascade of lattice structures, each associated with a lattice coefficient $\alpha_{i}$. They have two special characteristics:

-In each stage of the lattice, one coefficient is positive and the other one is negative, but both have the same magnitude. 
-All coefficients with even valued indices are zero.

The input output relations for the first and the further stages are given below:

$\left[\begin{array}{l}x_{L}^{(1)}(n) \\ x_{H}^{(1)}(n)\end{array}\right]=\left[\begin{array}{cc}1 & -\alpha_{1} \\ \alpha_{1} & 1\end{array}\right]\left[\begin{array}{c}x_{L}^{(0)}(n) \\ x_{H}^{(0)}(n-1)\end{array}\right]$

$\left[\begin{array}{l}x_{L}^{(2 m+1)}(n) \\ x_{H}^{(2 m+1)}(n)\end{array}\right]=\left[\begin{array}{cc}1 & -\alpha_{1} \\ \alpha_{1} & 1\end{array}\right]\left[\begin{array}{c}x_{L}^{(2 m-1)}(n) \\ x_{H}^{(2 m-1)}(n-2)\end{array}\right]$

with $x_{L}^{(0)}(n)=x_{H}^{(0)}(n)=x(n)$, where $x_{L}^{(2 m+1)}(n)$, and $x_{H}^{(2 m+1)}(n)$, are the lowpass (L) and highpass (H) output of the lattice filter at stage $(2 m+1)$, respectively.

The 1D PR Lattice filter structure defined above has to be optimized to determine the lattice filter parameters. To achieve this, in 1D frequency domain, the stopband energy is minimized at specific frequencies [12]. Lattice coefficients are obtained by the minimization of the stopband energy given as,

$$
\hat{\alpha}=\operatorname{argmin}_{\alpha}\left\|H_{L}^{(2 m+1)}\left(e^{j w}\right)\right\|, w_{s}<w<\pi
$$

where $H_{L}^{(2 m+1)}\left(e^{j w}\right)$, is the transfer function of the lattice filter at stage $(2 m+1)$, and $w_{s}$ is the desired stopband frequency. Since the lowpass and highpass filters of the lattice filters are mirror filters, the minimization of the object function will provide the corresponding lowpass and highpass filters.

\section{UNDECIMATED LATTICE SUB BAND DECOMPOSITION/ RECONSTRUCTION \\ 3.1 Proposed Undecimated Decomposition with Lattice Structure}

Since the decimated algorithm lacks the property of shiftinvariance, its performance will be affected by the shift of the input image which will be a severe drawback for the applications such as fusion, denoising and segmentation/classification. In undecimated wavelet transform, the down and upsampling processes are suppressed and at each decomposition level zeroes are inserted between the filter coefficients [9]. Following a similar strategy, we suppress the down and upsampling processes, and (1) and (2) is replaced by

$\left[\begin{array}{l}x_{L}^{(1)}(n) \\ x_{H}^{(1)}(n)\end{array}\right]=\left[\begin{array}{cc}1 & -\alpha_{1} \\ \alpha_{1} & 1\end{array}\right]\left[\begin{array}{c}x_{L}^{(0)}(n) \\ x_{H}^{(0)}\left(n-2^{l-1}\right)\end{array}\right]$

$\left[\begin{array}{l}x_{L}^{(2 m+1)}(n) \\ x_{H}^{(2 m+1)}(n)\end{array}\right]=\left[\begin{array}{cc}1 & -\alpha_{1} \\ \alpha_{1} & 1\end{array}\right]\left[\begin{array}{c}x_{L}^{(2 m-1)}(n) \\ x_{H}^{(2 m-1)}\left(n-2^{l}\right)\end{array}\right]$

where, $l=1,2, \ldots, L$ is the decomposition level.

Analysis and synthesis lattice structures used in the single level decomposition and reconstruction for 1D signals are shown in Fig.1.a and Fig.1.b, respectively. As seen in Fig 1.a the analysis lattice structure divides the input signal into its low-pass and high-pass components. The synthesis structure reconstructs the signal from these components. In Table 1, the parameters determined for different stopband frequencies according to (3) and corresponding average stopband powers

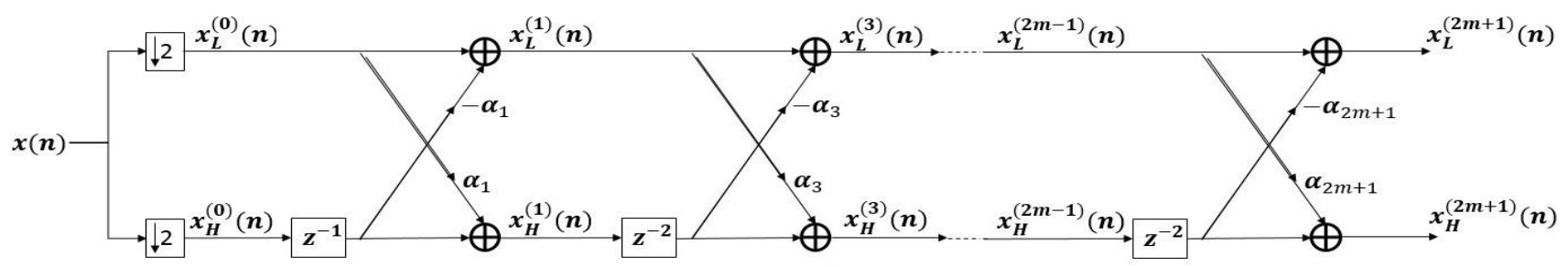

a)

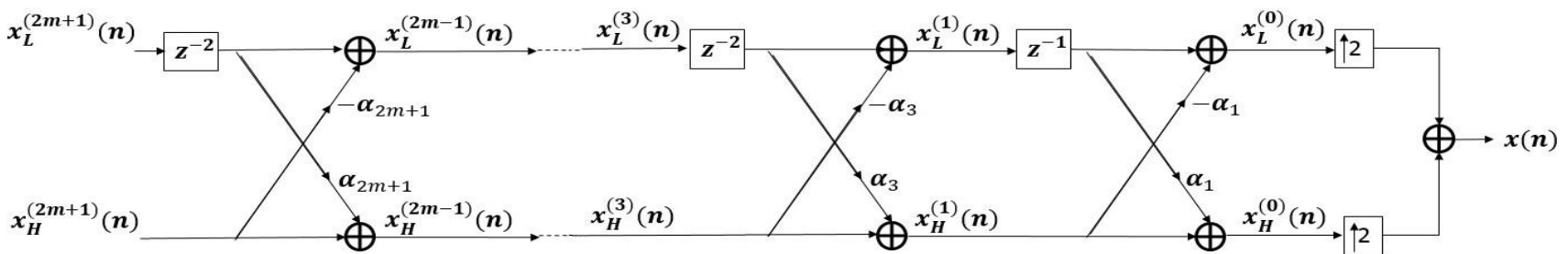

b)

Fig. 1. Undecimated 1-D a) Analysis Structure (Subband Decomposition) b) Synthesis Structure (Reconstruction)

are shown. One can observe that, the average stopband power decreases with the increase in the lattice order, as expected.
Table 1. Lattice coefficients

\begin{tabular}{|l|l|c|c|}
\hline $\begin{array}{l}\text { Stopband } \\
\text { Frequency }\end{array}$ & \multicolumn{2}{|l|}{$\begin{array}{l}\text { Lattice } \\
\text { Parameters }\end{array}$} & $\begin{array}{l}\text { Stopband Average } \\
\text { Power }\end{array}$ \\
\hline \multirow{4}{*}{$\mathbf{0 . 2 5} \boldsymbol{\pi}$} & $\alpha_{1}$ & -1 & 0.1415 \\
\cline { 2 - 4 } & $\alpha_{3}$ & 0.060944 & 0.0247 \\
\cline { 2 - 4 } & $\alpha_{5}$ & 0.000066 & 0.0005 \\
\hline
\end{tabular}


In order to perform image decomposition, the structure given in Fig.1.a is applied first to the rows, then to the columns of the image, or vice versa. The visual representation is given in Fig.2. In order to carry on the decomposition, the process given in Fig.2.a. is applied on the LL subband of the image.

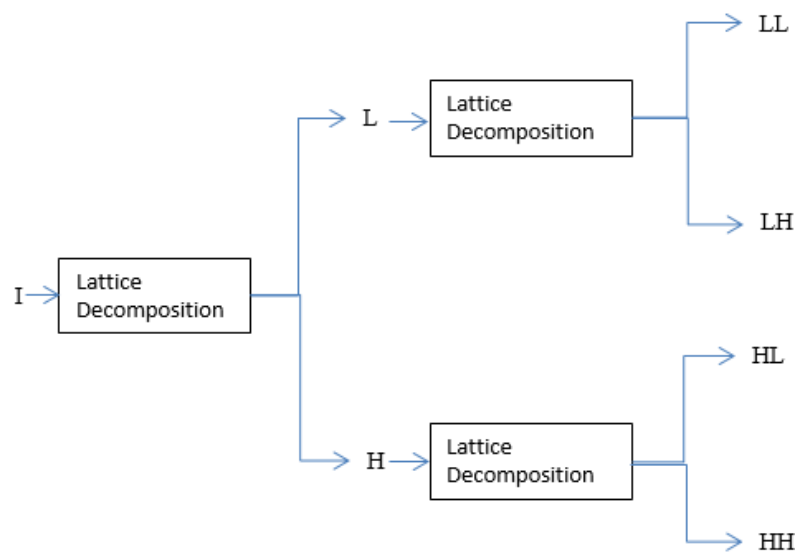

a)

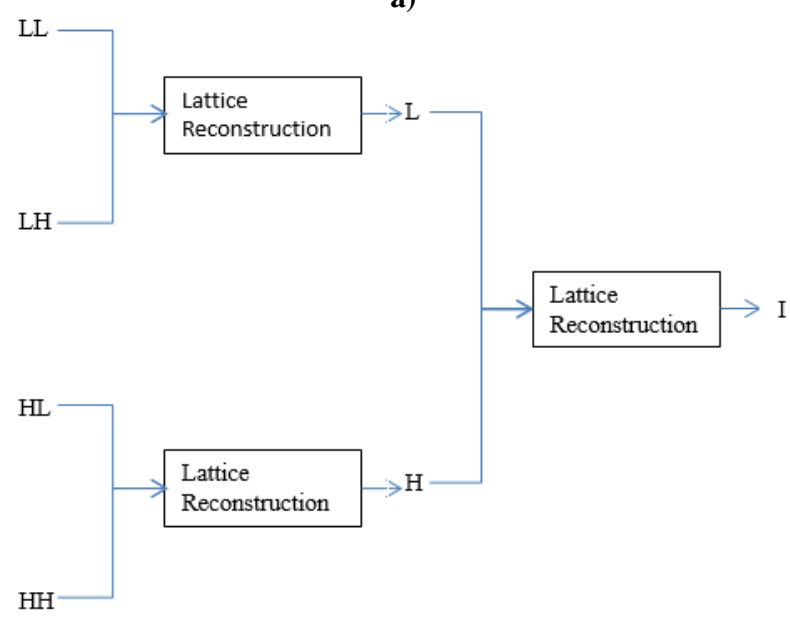

b)

Fig. 2. a) Image Decomposition and b) Reconstruction

\subsection{Fusion Process}

It is assumed that MS and PAN images have already been geometrically registered to each other. The proposed fusion methods may be summarized as in the following:

(1) Each band of the MS and PAN image are decomposed by the analysis lattice structure to obtain approximation and detail subbands.

(2) The approximation subband (LL) of the fused image is equal to the LL subband of the MS image

$$
F_{L L}=M S_{L L}
$$

(3) All detail subbands ( $\mathrm{LH}, \mathrm{HL}$ and $\mathrm{HH}$ ) of the MS and PAN images are merged by the following rule.

$$
\begin{aligned}
F_{L H} & =\max \left|M S_{L H}, P A N_{L H}\right| \\
F_{H L} & =\max \left|M S_{H L}, P A N_{H L}\right| \\
F_{H H} & =\max \left|M S_{H H}, P A N_{H H}\right|
\end{aligned}
$$

(4) One band of the fused image is reconstructed from the fused coefficients using synthesis lattice structure.
(5) The fusion process is repeated for the other bands of the MS image to obtain all the bands of the final fused image.

\section{EXPERIMENTAL RESULTS}

The proposed method is applied to the QuickBird MS and PAN images. The images are downloaded from http://glcf.umiacs.umd.edu/data/quickbird/. The area is Sundarbans, India and images are acquired on November 21, 2002. The spatial resolution of MS and PAN images are 2.8 and $0.7 \mathrm{~m}$, respectively. Bicubic interpolation is used for the registration of MS images. In order to compare the results with the original MS image, the MS and PAN images are spatially degraded by lowpass filtering and decimation to obtain $11.2 \mathrm{~m}$ resolution MS and $2.8 \mathrm{~m}$ resolution PAN images. The degraded MS and PAN images are fused by different methods and the fusion results were compared with the original MS images with $2.8 \mathrm{~m}$. resolution. The experiments were carried out with the proposed decimated and undecimated lattice methods and the resulting images obtained using discrete wavelet transform (DWT) and stationary wavelet transform (SWT) are also included for comparison. Simulations have been carried on for different decomposition levels and best results have been obtained for a decomposition level of 2 . The lattice filter used in the decomposition and reconstruction steps of our method is given in Table 1.

\subsection{Visual Analysis}

Spatially degraded $2.8 \mathrm{~m}$ resolution PAN, $11.2 \mathrm{~m}$. resolution MS and original $2.8 \mathrm{~m}$ resolution MS images are shown in Fig. 3 and Fig.4.a, b and c respectively. Fig. 3 and Fig. 4 d-h, show the fusion results obtained via G-IHS, DWT, SWT, decimated lattice (DL) and undecimated lattice (UDL) methods, respectively. The green squares is the zoomed versions of the red squares.

It can be concluded from the resulting images that the spatial resolution of the initial MS images appear to have been enhanced. They contain the structural details of the PAN images. GIHS provides the sharpest results, however color distortion can be easily observed in both of the images. The decimated cases, DWT and decimated lattice results present artifacts due to blocking effect. Undecimated methods, SWT and undecimated lattice provide better results. However, the result of SWT in Fig.4, loses some of the color information especially, around the lake, whereas UDL method preserves color information better in that area. The overall detail injection of two methods are similar. For Fig.3, undecimated lattice result is slightly better than SWT, around the zoomed area.

\subsection{Quantitative Comparison}

The comparison of the different fusion methods is performed quantitatively using the following indicators [14-18].

Correlation coefficient (CC) between each band of the original MS image and fused (F) image is defined as [14], 


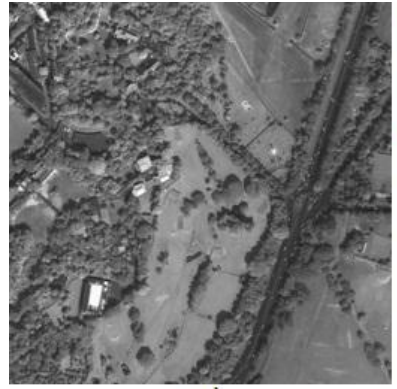

a)

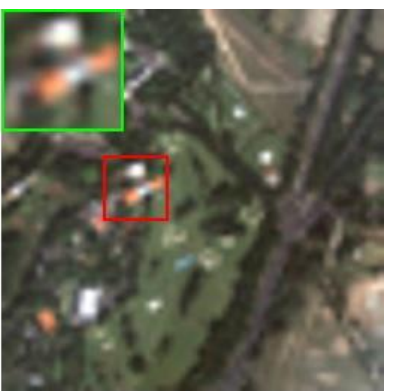

b)

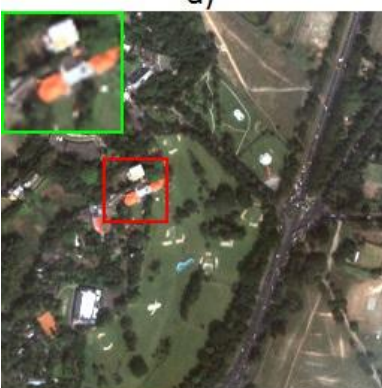

c)

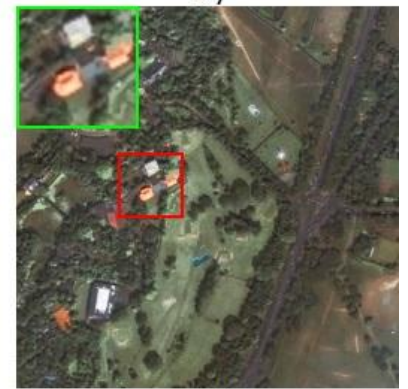

d)

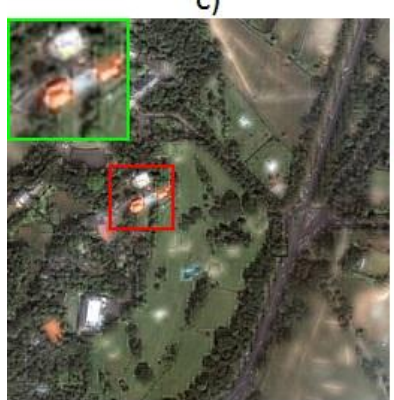

e)
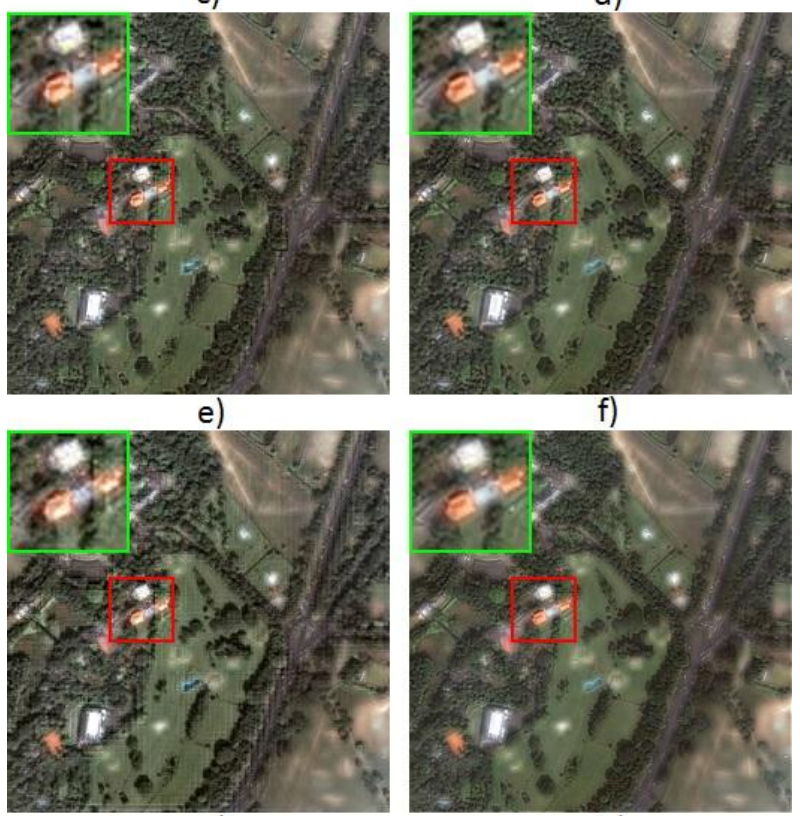

g) f)

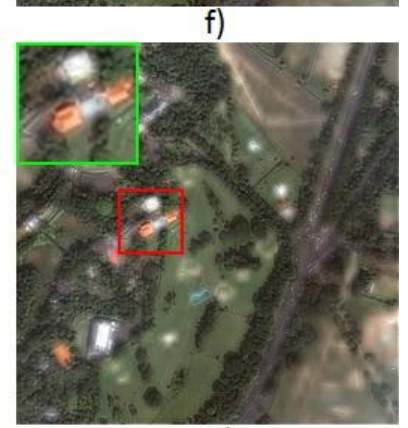

h)

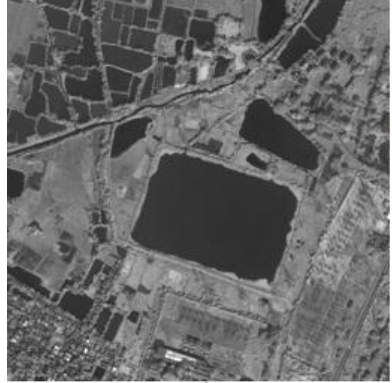

a)

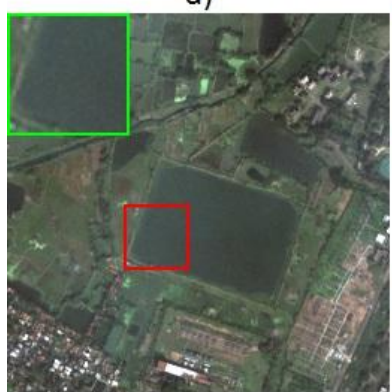

c)

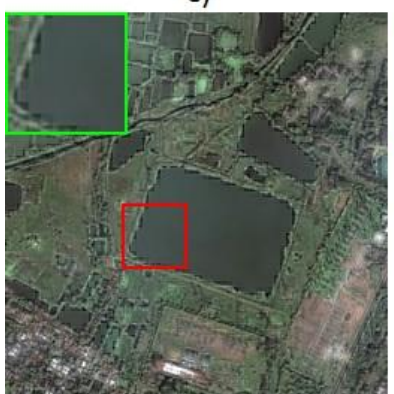

e)

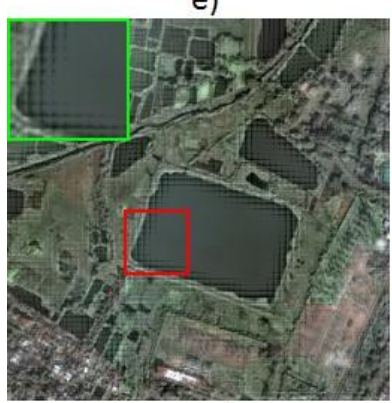

g)

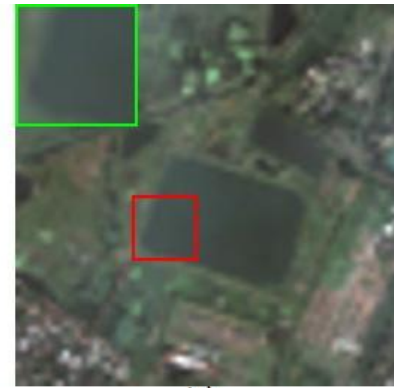

b)

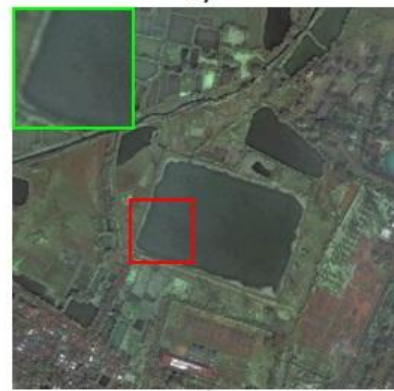

d)

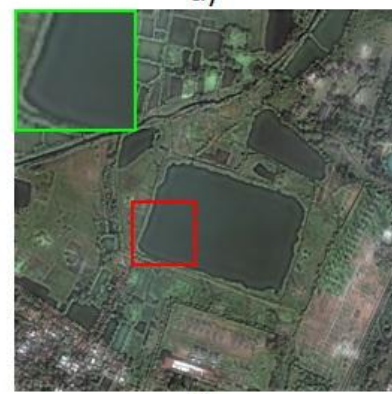

f)

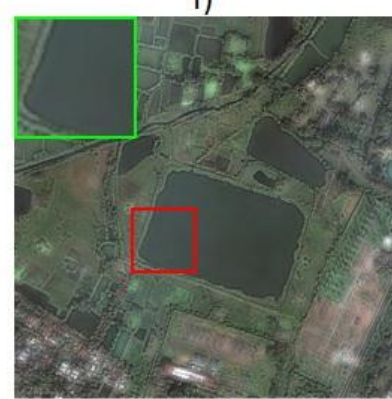

h)

Fig. 3. a) Degraded PAN 256*256), b) Degraded MS image $(256 * 256)$, c) original MS image $(256 * 256)$, fusion results for d) G-IHS e) DWT f) SWT g) DL, h) UDL

$C C=\frac{\sum_{m=1}^{M} \sum_{n=1}^{N}\left(M S(m, n)-\mu_{M S}\right)\left(F(m, n)-\mu_{F}\right)}{\sqrt{\sum_{m=1}^{M} \sum_{n=1}^{N}\left(M S(m, n)-\mu_{M S}\right)^{2} \sum_{m=1}^{M} \sum_{n=1}^{N}\left(F(m, n)-\mu_{F}\right)^{2}}}$

where $\mu_{M S}$ and $\mu_{F}$ are the mean values of the MS images and the fused image, respectively.

$\mathrm{CC}$ is a similarity criteria for images, having values between 1 and +1 . + 1 means two images are same, -1 means that images are opposite. $\mathrm{CC}$ within the bands of the original MS data and the fused data should be as close to 1 as possible to guarantee the preservation of the spectral content of the multispectral image. Table 4 and 5 show that higher CC values are obtained for additive lattice.

Fig. 4. a) Degraded PAN (256*256), b) Degraded MS image $(256 * 256)$, c) original MS image $(256 * 256)$, fusion results for d) G-IHS e) DWT f) SWT g) DL, h) UDL

RMSE (root mean square error) between each band of the original and the fused images can be defined as [14],

$R M S E=\sqrt{\frac{1}{M N} \sum_{m=1}^{M} \sum_{n=1}^{N}|M S(m, n)-F(m, n)|^{2}}$

RMSE provides a measure of the radiometric distortion of the fused image from the original MS image and should be as close to 0 as possible. RMSE values observed from Table 4 and 5 indicate that the pixel values are less distorted in additive lattice method.

SAM (spectral angle mapper) yields a global measurement of the spectral distortion and is defined as [15], 
$S A M=\arccos \left(\frac{\langle M S, F\rangle}{\|M S\|_{2}\|F\|_{2}}\right)$

where $M S$ and $F$ stand for the spectral vectors of the resampled MS and fused band. It is averaged over the whole image and should be as close to 0 as possible.

The Relative Global Dimensional Synthesis Error (ERGAS) [16] is defined as,

$E R G A S=100 \frac{h}{l} \sqrt{\frac{1}{N} \sum_{i=1}^{N} \frac{R M S E^{2}\left(M S_{i}, F_{i}\right)}{M_{i}^{2}}}$

where $h$ and $l$ are the resolution of high and low spatial resolution images, respectively. $M_{i}$ is the mean radiance of $i$ th band.

The lower values of the ERGAS indexes indicate higher spectral quality of the fused images.

The Spatial ERGAS (SERGAS) [17] is defined as,

$S E R G A S=100 \frac{h}{l} \sqrt{\frac{1}{N} \sum_{i=1}^{N} \frac{R M S E^{2}\left(P A N_{i}, F_{i}\right)}{M_{i}^{2}}}$

where $P A N_{i}$ is the PAN image, whose histogram is matched to the ith band of the MS image. The lower values of the SERGAS indexes indicate higher spatial quality of the fused images.

Q-average (Universal Image Quality Index) [18] models any distortion between two images as a combination of loss of correlation, luminance distortion and contrast distortion.

$U I Q=\frac{\sigma_{M S F}}{\sigma_{M S} \sigma_{F}} \frac{2 \mu_{M S} \mu_{F}}{\mu_{M S+}^{2} \mu_{F}^{2}} \frac{\sigma_{M S} \sigma_{F}}{\sigma_{M S+}^{2} \sigma_{F}^{2}}$

Q-average is defined as the average value of the UIQ's of each band. A higher value indicates better quality.

The quantitative comparisons of Fig. 3 and Fig. 4 are given in Table 2 and Table 3, respectively.

As it is expected, GIHS result has the best SERGAS value, which means that, GIHS keeps the spatial information of PAN image the most. However, other metrics show that, the GIHS results are spectrally distorted. Decimated cases of lattice and SWT, even though having better spectral metric scores than GIHS method, cannot preserve the spectral information good enough. The best spectral results are achieved by the proposed undecimated lattice method, followed by the SWT method. The quantitative comparisons show that, the proposed method is better than SWT.

Table 2. Quantitative comparison of the fusion methods for the image shown in Fig.3

\begin{tabular}{|c|c|c|c|c|c|c|}
\hline \multicolumn{2}{|l|}{ Index } & GIHS & DWT & SWT & DL & UDL \\
\hline \multirow{4}{*}{$\mathrm{CC}$} & $\mathbf{R}$ & 0.7926 & 0.9001 & 0.9188 & 0.8662 & 0.9206 \\
\hline & G & 0.7794 & 0.9137 & 0.9280 & 0.8855 & 0.9297 \\
\hline & B & 0.7229 & 0.8731 & 0.8972 & 0.8240 & 0.9047 \\
\hline & NIR & 0.8532 & 0.9185 & 0.9161 & 0.9076 & 0.9075 \\
\hline \multirow{3}{*}{ RMSE } & $\mathbf{R}$ & 26.3107 & 18.8277 & 16.9429 & 22.1790 & 16.7698 \\
\hline & G & 25.0536 & 16.3551 & 14.9349 & 19.2693 & 14.9171 \\
\hline & B & 31.1683 & 22.4535 & 20.0230 & 27.3792 & 19.2265 \\
\hline
\end{tabular}

\begin{tabular}{|c|c|c|c|c|c|}
\hline NIR & 9.3414 & 7.1114 & 7.2632 & 7.5312 & 7.5270 \\
\hline ERGAS & 6.0964 & 4.2879 & 3.8566 & 5.1075 & 3.8121 \\
\hline SERGAS & 4.2499 & 7.8312 & 7.8620 & 7.3704 & 8.0136 \\
\hline SAM & 4.6563 & 4.2029 & 3.7314 & 5.3054 & 3.4624 \\
\hline Q-average & 0.7236 & 0.8275 & 0.8506 & 0.7860 & 0.8528 \\
\hline Time (sec) & 0.272 & 0.314 & 1.490 & 0.256 & 1.065 \\
\hline
\end{tabular}

Table3. Quantitative comparison of the fusion methods for the image shown in Fig.4

\begin{tabular}{|c|c|c|c|c|c|c|}
\hline \multicolumn{2}{|l|}{ Index } & \multirow{2}{*}{\begin{tabular}{|l|} 
GIHS \\
0.6986
\end{tabular}} & \multirow{2}{*}{$\begin{array}{l}\text { DWT } \\
0.7756\end{array}$} & \multirow{2}{*}{$\begin{array}{l}\text { SWT } \\
0.8154\end{array}$} & \multirow{2}{*}{$\begin{array}{l}\text { DL } \\
0.6934\end{array}$} & \multirow{2}{*}{\begin{tabular}{|l|} 
UDL \\
0.8423
\end{tabular}} \\
\hline \multirow{4}{*}{$\mathrm{CC}$} & $\mathbf{R}$ & & & & & \\
\hline & G & 0.7174 & 0.8119 & 0.8465 & 0.7441 & 0.8645 \\
\hline & B & 0.6214 & 0.7249 & 0.7710 & 0.6299 & 0.8049 \\
\hline & NIR & 0.9420 & 0.9577 & 0.9609 & 0.9533 & 0.9493 \\
\hline \multirow{4}{*}{ RMSE } & $\mathbf{R}$ & 20.1469 & 19.5732 & 17.1430 & 25.1532 & 15.3935 \\
\hline & G & 17.1368 & 15.7524 & 13.6789 & 20.3790 & 12.4695 \\
\hline & B & 20.5417 & 20.6327 & 17.9927 & 26.8186 & 15.9572 \\
\hline & NIR & 9.0083 & 7.7694 & 7.5017 & 8.1742 & 8.3598 \\
\hline \multicolumn{2}{|c|}{ ERGAS } & 4.3350 & 4.1783 & 3.6679 & 5.3532 & 3.3470 \\
\hline \multicolumn{2}{|c|}{ SERGAS } & 3.4575 & 5.4740 & 5.3513 & 5.3097 & 5.2878 \\
\hline \multicolumn{2}{|l|}{ SAM } & 4.0413 & 4.4545 & 4.0304 & 6.0165 & 3.7021 \\
\hline \multicolumn{2}{|c|}{ Q-average } & 0.6167 & 0.6659 & 0.7158 & 0.5836 & 0.7518 \\
\hline \multicolumn{2}{|c|}{ Time (sec) } & 0.278 & 0.322 & 1.572 & 0.261 & 1.154 \\
\hline
\end{tabular}

Taking into consideration both visual and quantitative analysis, proposed undecimated lattice method outperforms all the considered wavelet based methods. In addition, the time spent for the methods are given in the bottom row of Table 2 and Table 3. The methods are realized in a PC of Intel Core i3 $3.06 \mathrm{GHz}$ and $4 \mathrm{~GB}$ of Rams and the results show that the proposed undecimated lattice structure is faster than SWT method.

\section{CONCLUSION}

A new pansharpening method based on the decomposition of the source images via QMF filterbanks with undecimated lattice structure is proposed. The decomposed images are merged in the subband domain using a predefined rule and the fused image is obtained by the reconstruction filters. The new structure unifies the entire decomposition/reconstruction step into a single structure as lattice analysis/synthesis structure. The approximation and detail images are simultaneously obtained using simple mathematical operations. The fusion results confirm that lattice filterbanks based method preserves better the spectral characteristics of the MS images while increasing the spatial resolution compared to the other stateof-the-art pansharpening methods. Since the proposed method has similar decomposition/reconstruction steps as wavelet decomposition with decreased computation time and better fusion results, it can be a good alternative to the wavelet transform for pansharpening purposes. 


\section{REFERENCES}

[1] Piella, G. 2002. A General framework for multiresolution image fusion: from pixels to regions. Information Fusion, 4(4), 1386-3711.

[2] Pohl, C. and Van Genderen J. L. 1998. Multisensor image fusion in remote sensing: concepts, methods and applications. Int. Journal of Remote Sensing, 99(5), 823854.

[3] Chavez, P. S., Stuart, J., Sides, C. and Anderson J. A. 1991. Comparison of three different methods to merge multiresolution and multispectral data: Landsat TM and SPOT panchromatic. Photogrammetric Engineering and Remote Sensing, 57(14), 259-303.

[4] Carper, W. J., Lilesand, T. W. and Kieffer, R.W. 1990. The use of Intensity-Hue-Saturation transformation for merging SPOT panchromatic and multispectral image data. Photogrammetric Engineering and Remote Sensing, 56(4), 459-467.

[5] Tu, T. M., Huang, P. S., Huang, C. L. and Chang, C. P. 2004. A fast intensity-hue-saturation fusion technique with spectral adjustement for IKONOS imagery. IEEE Trans. Geosci. Remote Sens. Lett., 1(4), 309-312.

[6] Choi, M. 2006.A new intensity-hue-saturation approach to image fusion with a tradeoff parameter. IEEE Trans. Geosci, Remote Sens., 44(6), 1672-1682.

[7] Chavez, P. S. and Kwarteng, A.Y. 1989. Extracting spectral contrast in Landsat Thematic Mapper image data using selective principal component analysis. Photogrammetric Engineering and Remote Sensing, 55, 339-348.

[8] Mallat, S.G. 1989. A theory for multiresolution signal decomposition: The wavelet representation. IEEE Trans. Pattern Anal. Machine Intell., 11(7), 674-693.

[9] Pajares, G. and dela Cruz, J. M. 2004. A wavelet-based image fusion tutorial. Pattern Recognition, 37(9), 18551872 .
[10] Li, S., Kwok, J. T. and Wang, Y. 2002. Using The discrete wavelet frame transform to merge Landsat TM and SPOT panchromatic images. Information Fusion, $3(1), 17-23$

[11] Li, S., Yang, B. and Hu, J. Performance comparison of different multiresolution transforms for image fusion. Information Fusion, 12(2), 74-84.

[12] Vaidyanathan, P. and Hoang, P-Q. 1988. Lattice structures for optimal design and robust implementation of two-channel perfect reconstruction QMF Banks. IEEE Trans. ASSP, 36(1), 81-94.

[13] Sezen, S. and Ertüzün, A. 2006. 2D Four-channel perfect reconstruction filter bank realized with the $2 \mathrm{D}$ lattice filter structure. EURASIP Journal on Applied Signal Processing, 2006, 1-16.

[14] Eskicioglu, A. and Fisher, P. 1995. Image Quality measures and their performance. IEEE Trans. On Comm., 43(12), 2959-2965.

[15] Alperone, L., Wald, L., Chanussot, J., Thomas, C., Gamba, P. and Mann Bruce, L. 2007. Comparison of pansharpening algoritms: outcome of the 2006 GRS-S data-fusion contest. IEEE trans Geosci. Remote Sens. 45(10), 3012-3021.

[16] Wald, L., Ranchin, T. and Mangolini, M. 1997. Fusion of satellite images of different spectral resolutions: Assessing the quality of resulting images. Photogramm. Eng. Remote Sens., 63(6), 691-699.

[17] Lillo-Saavedra, M., Gonzalo, C., Arquero, A. and Martinez, E. 2005. Fusion of multispectral and panchromatic satellite sensor imagery based on tailored filtering in the Fourier domain. International Journal of Remote Sensing. 26(6), 1263-1268.

[18] Bovik, A. and Wang, Z. 2002. A universal image quality index. IEEE Signal Proces. Lett, 9(3), 81-84. 